\title{
Recent Progress in Jellyfish Toxin Study
}

\author{
Hiroshi Nagai* \\ Department of Ocean Sciences, Tokyo University of Marine Science and Technology, 4-5-7 Konan, Minato-ku, Tokyo 108-8477, Japan
}

(Received June 6, 2003)

\begin{abstract}
Recently, we have reported the chemical properties of some jellyfish proteinaceous toxins. These were the first chemical characterizations of jellyfish protein toxins to be reported. The isolation of the proteinaceous toxins in their active forms was the key step in the studies. We isolated the toxins from three box jellyfish (Cubozoa) species [Carybdea rastoni (C. rastoni), Carybdea alata (C. alata), and Chiropsalmus quadrigatus (C. quadrigatus)]. These toxins showed lethal toxicity to crustaceans and hemolytic activity. Furthermore, the full-length cDNAs and the deduced amino acid sequences of the toxins were clarified. All of these toxins have a molecular weight of around $45 \mathrm{kDa}$ and their amino acid sequences showed homology with each other. The box jellyfish toxins represent a novel bioactive protein family.
\end{abstract}

Key words — jellyfish, protein, toxin, Cubozoa, sting

\section{INTRODUCTION}

Some jellyfish have venomous stings and are harmful to humans. For example, the deadly box jellyfish Chironex fleckeri (C. fleckeri) has caused many fatalities in Australia and the Portuguese manof-war Physalia physalis also has a severe sting occasionally causing death. ${ }^{1)}$ From the public health point of view, jellyfish toxins have been extensively examined and revealed to be mostly proteinaceous toxins. ${ }^{2)}$ However, the precise chemical nature of none of the jellyfish toxins has been successfully elucidated, because the lability of these toxins has hampered further studies. ${ }^{3)}$ Among the many jellyfish species, box jellyfish (Cubozoa) are especially recognized as hazardous and include $C$. fleckeri, Chiropsalmus quadrigatus (C. quadrigatus), Carukia barnesi, Carybdea rastoni (C. rastoni), and Carybdea alata (C. alata). ${ }^{4}$ Thus our group has focused on the chemical characterization of box jellyfish toxins. This paper summarizes the recent progress in the study of jellyfish toxin, especially box jellyfish toxin.

\footnotetext{
*To whom correspondence should be addressed: Department of Ocean Sciences, Tokyo University of Marine Science and Technology, 4-5-7 Konan, Minato-ku, Tokyo 108-8477, Japan. Tel.: +81-3-5463-0454; Fax: +81-3-5463-0398; E-mail: nagai@edu. s.kaiyodai.ac.jp
}

\section{Proteinaceous Toxins of $\boldsymbol{C}$. rastoni}

The box jellyfish (Sea Wasp) C. rastoni (andonkurage in Japanese) is one of the most annoying marine organisms for swimmers and bathers at the Japanese seashore. ${ }^{5)}$ The stinging of $C$. rastoni causes cutaneous pain and inflammation in humans. Thus we choose $C$. rastoni as the first target of study. During that study, we observed that the toxicity of the proteinaceous toxins from $C$. rastoni was markedly decreased during purification, storage, and sample concentration. Determining the optimal conditions for the purification and storage of the toxins was the key step in characterizing them. It was found that ion-exchange chromatography, but not an ultrafiltration system, was suitable for concentrating toxins. We also found that the purified samples should be stored in a high salt-concentration solution, and should not be frozen or freeze-dried. Thus we stored the purified toxins in a solution with a high salt concentration such as $0.8 \mathrm{M} \mathrm{NaCl}$ and $5 \mathrm{mM}$ phosphate buffer $\left(\mathrm{pH} \mathrm{6.0)}\right.$ ) at $4^{\circ} \mathrm{C}$. Under this condition, $90 \%$ of the hemolytic activity of $C$. rastoni toxins could be retained for more than 6 months of storage. Finally, two proteinaceous toxins, $C$. rastoni toxins A and B (CrTX-A, 43 kDa; CrTX-B, 46 kDa), were isolated in their active forms from the tentacle of the box jellyfish $C$. rastoni. Peptide mapping revealed some amino acid sequences of the peptide fragments originating from the toxins. Based on the 


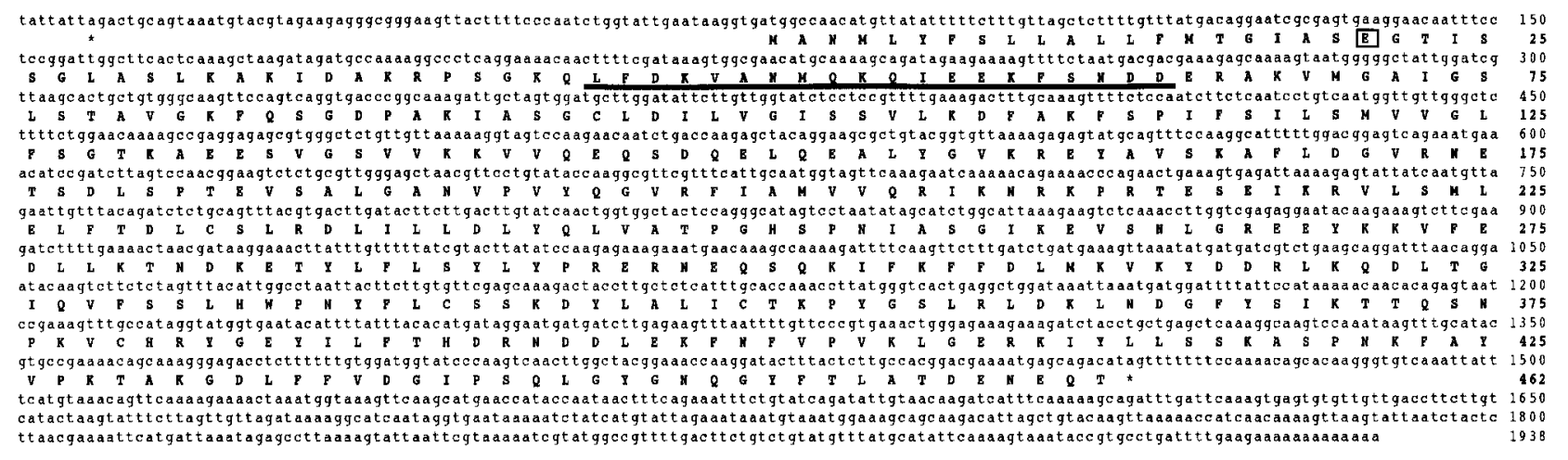

Fig. 1. Nucleotide and Amino Acid Sequences of CqTX-A

The complete sequence of CqTX-A cDNA and its translation product are illustrated. The deduced amino acid sequence is shown from the first ATG codon of the open reading frame. The asterisks indicate in-frame stop codons (TAG). Nucleotide and amino acid numbers are shown at the right. The underlined sequence has an amphiphilic $\alpha$-helix-like sequence (Fig. 2). A box indicates the $N$-terminal amino acid of the mature CqTX-A.

amino acid sequence information, degenerate RTPCR and 5' - and 3'-rapid amplification of cDNA ends (RACE) experiments were performed that finally revealed the $5^{\prime}$ - and $3^{\prime}$-termini sequences of the full-length cDNA (1600 bp) that encodes both CrTX-A and CrTX-B. Histopathologic studies, clearly showed that CrTX-A $(0.1 \mu \mathrm{g} /$ injection $)$ caused inflammation of the affected area comparable to the symptoms in humans after $C$. rastoni stings. CrTX-A was the main toxin in the nematocyst, which is the organ responsible for cnidarian stinging. Thus the results of pathologic study showed CrTX-A was the toxin responsible for the cutaneous inflammation observed in humans after $C$. rastoni stings. CrTX-A was the first toxin isolated that was determined to be the causative agent of cutaneous inflammation due to jellyfish stings.

\section{Proteinaceous Toxins from $C$. alata}

The box jellyfish (sea wasp) C. alata is a stinging jellyfish. ${ }^{6}$ Stinging by $C$. alata causes cutaneous pain and inflammation in humans, similar to that by $C$. rastoni. This species is distributed widely in the tropical zones of the Atlantic, Pacific, and Indian Oceans. Interestingly, in Hawaii, massive invasions by this species occur every 9 th or 10 th day after a full moon on the southern shores (Waikiki Beach) of Oahu. ${ }^{7)}$ C. alata toxins A and B (CaTX$\mathrm{A}, 43 \mathrm{kDa}$; CaTX-B, $45 \mathrm{kDa}$ ) were successfully isolated employing the toxin isolation procedure developed for $C$. rastoni mentioned above. We found that CaTX-A, but not CaTX-B, was present in $C$. alata nematocysts. The full-length cDNA (2000 bp) that encodes a 463 -amino acid protein (CaTX-A) was determined.

\section{Proteinaceous Toxins from C. quadrigatus}

The box jellyfish (sea wasp) C. quadrigatus (habu-kurage in Japanese) is widely distributed in the tropical Indo-Pacific region. ${ }^{8)}$ The sting of $C$. quadrigatus is so severe that it is sometimes fatal to humans. In Okinawa, a tropical region of Japan, three fatal stings have been officially reported so far. ${ }^{9)}$ Death is caused by cardiac arrest in systole or respiratory failure with acute pulmonary edema. Children are particularly susceptible.

C. quadrigatus toxin A (CqTX-A, $44 \mathrm{kDa})$ was isolated using the toxin isolation procedure developed for the study of $C$. rastoni toxin. Furthermore, the isolation procedure for CqTX-A could be simplified in comparison with the procedures for $C$. rastoni and $C$. alata toxins because the cleanly isolated nematocysts were used as a starting material. The full-length cDNA (1938 bp) that encodes a 462amino acid proteinaceous toxin (CqTX-A) was determined (Fig. 1).

\section{Comparison of Proteinaceous Toxins Obtained from Box Jellyfish}

The calculated isoelectric points for these proteinaceous toxins from box jellyfish indicated the basic nature of these proteins. During the study of toxins from $C$. rastoni, remarkable loss of sample toxicity during storage or purification were caused by adhesion of the toxins to the glass or membrane filter. Furthermore, we could not recover active toxins from the glass or membrane filter. Thus the basic nature of these protein toxins may account for the adhesive tendency and also the instability of the box jellyfish toxins.

Comparison of the deduced CaTX-A amino acid 


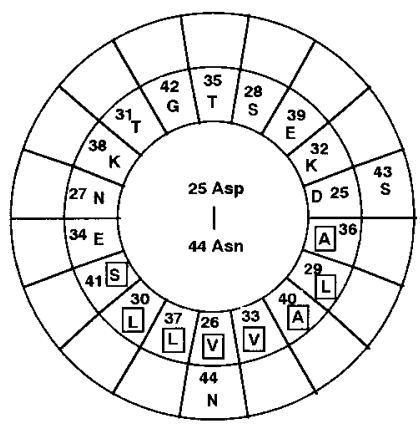

CrTXs

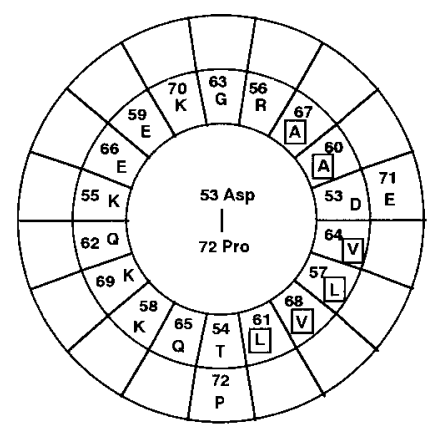

CaTX-A

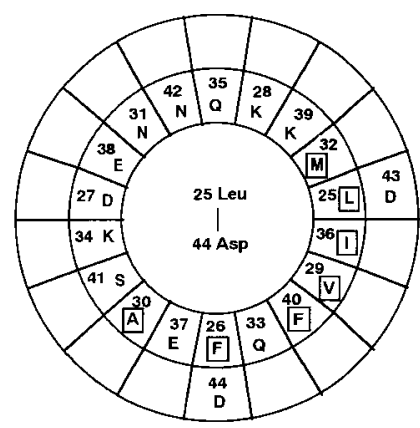

CqTX-A

Fig. 2. Predicted Amphiphilic $\alpha$-Helices in CrTXs, CaTX-A, and CqTX-A

The potential amphiphilic $\alpha$-helices were predicted in the structures of CrTXs, CaTX-A, and CqTX-A. They are depicted by Edmundson helical wheels. ${ }^{10)}$ Hydrophobic amino acids are boxed. CrTXs, from aspartic acid (Asp)-25 to asparagine (Asn)-44; CaTX-A, from Asp-53 to proline (Pro)-72; CqTX-A, from leucine (Leu)-25 to Asp-44.

Table 1. Comparison of Lethal Toxicity and Hemolytic Activity of the Box Jellyfish Toxins

\begin{tabular}{lcc}
\hline \hline Toxin (Organism) & $\begin{array}{l}\text { Lethal toxicity to crayfish } \\
\text { (intraperitoneal, } \mathrm{LD}_{50} \text { value) }\end{array}$ & $\begin{array}{l}\text { Hemolytic activity } \\
(0.8 \% \text { sheep red blood cells, EC } 50 \text { value })\end{array}$ \\
\hline CrTX-A $($ C. rastoni) & $5 \mu \mathrm{g} / \mathrm{kg}$ & $2 \mathrm{ng} / \mathrm{ml}$ \\
CaTX-A $($ C. alata $)$ & $5-25 \mu \mathrm{g} / \mathrm{kg}$ & $70 \mathrm{ng} / \mathrm{ml}$ \\
CqTX-A $($ C. quadrigatus $)$ & $80 \mu \mathrm{g} / \mathrm{kg}$ & $160 \mathrm{ng} / \mathrm{ml}$ \\
\hline
\end{tabular}

sequence using the BLAST algorithm revealed homology (43.7\%) with that of CrTXs. ${ }^{6}$ The deduced CqTX-A amino acid sequence had $25.2 \%$ homology with that of CrTXs and $21.6 \%$ homology with that of CaTX-A. ${ }^{8)}$ Comparison of the box jellyfish toxin amino acid sequences with those of other proteins using the BLAST (basic local alignment search tool) algorithm showed no significant similarity. ${ }^{8)}$ Since a novel bioactive protein family has emerged from these studies of the box jellyfish toxins (CrTXs, CaTX-A, and CqTX-A), we propose here to designate these novel bioactive proteins as the box jellyfish toxin family. Secondary structural analysis of these toxins predicted the presence of $\alpha$-helices that existed in the $\mathrm{N}$-terminus region. Construction of an "Edmundson wheel" ${ }^{10)}$ showed that the predicted $\alpha$ helices were amphiphilic (Fig. 2). Previous studies suggested that the amphiphilic structures of some cytolytic protein toxins allow the formation of pores in cell membranes, ${ }^{11-13)}$ and thus the amphiphilic structures of the box jellyfish toxins may explain their potent hemolytic activity (Table 1).

\section{Lethal Toxicity and Hemolytic Activity}

The $\mathrm{LD}_{50}$ values (intraperitoneal injection) of CqTX-A, CrTX-A, and CaTX-A in crayfish
(Procambarus clarkii) were $80 \mu \mathrm{g} / \mathrm{kg}, 5 \mu \mathrm{g} / \mathrm{kg}$, and 5-25 $\mu \mathrm{g} / \mathrm{kg}$, respectively (Table 1 ). CqTX-A, CrTX$\mathrm{A}$, and CaTX-A caused $50 \%$ hemolysis in $0.8 \%$ sheep red blood cells at a concentration of $160 \mathrm{ng} /$ $\mathrm{ml}, 2 \mathrm{ng} / \mathrm{ml}$, and $70 \mathrm{ng} / \mathrm{ml}$, respectively (Table 1). ${ }^{5,6,8)}$ These data indicate that CqTX-A is less toxic than CrTX-A and CaTX-A. The number of tentacles of C. quadrigatus is several times greater than that of C. alata or C. rastoni. Furthermore, the length of the tentacles of $C$. quadrigatus is at least three times that of $C$. alata or $C$. rastoni. Therefore, in human stings, the amount of toxin injected into the victim by $C$. quadrigatus in one incident should be much greater than the amounts injected by $C$. alata or $C$. rastoni. This is one reason why $C$. quadrigatus stings are more dangerous than those of $C$. rastoni or $C$. alata. Massive regional stings were observed on the bodies at autopsy after fatal $C$. quadrigatus incidents. CqTX-A was the major toxin in the nematocysts, and thus CqTX-A is likely to be the causative toxin in fatal cases of $C$. quadrigatus stings. Further study on the mode of action of these toxins will lead to the development of effective and specific remedies for box jellyfish stings. 
Acknowledgements The author thanks Mr. N. Oshiro, Ms. S. Iwanaga, and Mr. M. Nozaki of the Okinawa Prefectural Institute of Health and Environment; Ms. K. Takuwa-Kuroda of the Suntory Institute for Bioorganic Research (SUNBOR); Dr. M. Nakao of Suntory Ltd.; Drs. E. Ito, M. Miyake, and M. Noda of Chiba University for research collaboration; and Dr. T. Nakajima of SUNBOR for encouragement. This research was supported by grants-inaid from the Ministry of Education, Science, Sports and Culture of Japan and by a SUNBOR grant.

\section{REFERENCES}

1) Fenner, P. J. and Williams, J. A. (1996) Worldwide deaths and severe envenomation from jellyfish stings. Med. J. Aust., 165, 658-661.

2) Walker, M. J. A. (1988) Coelenterate and Echinoderm toxins: mechanisms and actions. In Handbook of Natural Toxins (Tu, A. T., Ed.), Marcel Dekker, New York, vol. 3, pp. 279-325.

3) Bloom, D. A., Burnett, J. W. and Alderslade, P. (1998) Partial purification of box jellyfish (Chironex fleckeri) nematocyst venom isolated at the beachside. Toxicon, 36, 1075-1085.

4) Williamson, J. and Burnett, J. (1995) Clinical toxicology of marine coelenterate injuries. In Clinical Toxicology of Animal Venoms and Poisons (Meiter, J. and White, J., Eds.), CRC Press, Boca Raton, FL, pp. 89-116.

5) Nagai, H., Takuwa, K., Nakao, M., Ito, E., Miyake, M., Noda, M. and Nakajima, T. (2000) Novel proteinaceous toxins from the box jellyfish (sea wasp) Carybdea rastoni. Biochem. Biophys. Res. Commun., 275, 582-588.

6) Nagai, H., Takuwa, K., Nakao, M., Sakamoto, B., Crow, G. L. and Nakajima, T. (2000) Isolation and characterization of a novel protein toxin from the Hawaiian box jellyfish (sea wasp) Carybdea alata. Biochem. Biophys. Res. Commun., 275, 589-594.

7) Thomas, C. S., Scott, S. A., Galanis, D. J. and Goto, R. S. (2001) Box jellyfish (Carybdea alata) in Waikiki: Their influx cycle plus the analgesic effect of hot and cold packs on their stings to swimmers at the beach: a randomized, placebo-controlled, clinical trial. Hawaii Med.J., 60, 100-107.

8) Nagai, H., Takuwa-Kuroda, K., Nakao, M., Oshiro, N., Iwanaga, S. and Nakajima, T. (2002) A novel toxin from the deadly box jellyfish (sea wasp, habukurage) Chiropsalmus quadrigatus. Biosci. Biotechnol. Biochem., 66, 97-102.

9) Okinawa Prefectural Institute for Health and Environment (1999) Kaiyo Kiken Seibutsu Taisakujigyo Houkokusho, Okinawa, pp. 1-17 (in Japanese).

10) Schiffer, M. and Edmundson, A. B. (1967) Use of helical wheels to represent the structures of proteins and to identify segments with helical potential. Biophys. J., 7, 121-135.

11) Kini, R. M. and Evans, H. J. (1989) A common cytolytic region in myotoxins, hemolysins, cardiotoxins and antibacterial peptides. Int. J. Pept. Protein. Res., 34, 277-286.

12) Belmonte, G., Menestrina, G., Pederzolli, C., Krizaj, I., Gubensek, F., Turk, T. and Macek, P. (1994) Primary and secondary structure of a pore-forming toxin from the sea anemone, Actinia equina L., and its association with lipid vesicles. Biochem. Biophys. Acta, 1192, 197-204.

13) Ghadessy, F. J., Chen, D., Kini, R. M., Chung, M. C. M., Jeyaseelan, K., Khoo, H. E. and Yuen, R. F. (1996) Stonustoxin is a novel lethal factor from stonefish (Synanceja horrida) venom. cDNA cloning and characterization. J. Biol. Chem., 271, 2557525581. 